\title{
Numerical Investigation of Flow Control by Suction and Injection on a Subsonic Airfoil
}

\author{
${ }^{1}$ M.H. Shojaefard, ${ }^{1}$ A.R. Noorpoor, ${ }^{1}$ A. Avanesians and ${ }^{2}$ M. Ghaffarpour \\ ${ }^{1}$ Automotive Engineering Department, Iran University of Science and Technology, Tehran, Iran \\ ${ }^{2}$ Department of Mechanical Engineering, University of Illinois at Chicago \\ 842 Taylor Street, 2039 ERF (MC 251), Chicago, Illinois 60607
}

\begin{abstract}
This study presents a numerical study concerning flow control by suction and injection. The case studied is the flow field over a subsonic airfoil with four suction and injection slots on the suction side of the airfoil. Five different angles of attack, 0, 5, 10, 13.3 and 20 with the Mach number of 0.15 are studied. Three cases are studied in each angle of attack. The three cases are airfoil with surface suction, surface injection and the base airfoil. A commercial CFD code, the FLUENT, is used in this study. The effect of suction and injection on aerodynamic coefficients is investigated. The results show that the surface suction can significantly increase the lift coefficient. The injection decreases the skin friction.
\end{abstract}

Key words: Suction, injection, CFD, flow control

\section{INTRODUCTION}

The energy needed to overcome the frictional force on an airplane is the substantial part of the total energy consumed on flying the airplane. In a transport airplane flying at subsonic speeds and in cruise condition, approximately $50 \%$ of the energy (fuel) is used to overcome the skin friction of the boundary layer. The boundary layer is mostly turbulent on such airplanes. Turbulent boundary layer has more surface friction than laminar boundary layer. Thus, we tend to keep the flow laminar on the surface. This reduces skin friction. Also, the separation of the boundary layer is associated with large energy losses and in most applications adversely affects the aerodynamic loads in the form of lift loss and drag increase. Therefore, there is a strong tendency to delay or manipulate the occurrence of flow separation. Hence, separation control is of great importance to most of the systems involving fluid flow, such as air, land or underwater vehicles and turbomachinary.

In the case of an external flow, such as the flow of the exterior surface of an aircraft, the objective is to delay the transition from laminar to turbulent, to suppress turbulence and to prevent separation. To do this we need to control the flow. The results include drag reduction, lift enhancement and flow-induced noise suppression. By controlling the flow, the fuel burned might be decreased almost 30 percent as reported by Braslow ${ }^{[1]}$. As a result, the pollutant emissions are reduced. In addition, lower fuel consumption will reduce the operating costs of commercial airplanes at least $8 \%{ }^{[1]}$.

To control the flow, passive or active devices are used. Passive control devices are those, which are not energy consumptive. They mainly affect the flow by the geometry of the airfoil. In contrast, active control devices use energy such as surface suction or injection. Natural laminar flow implies delaying transition via controlling the body shape to provide long runs of favorable pressure-gradient. This has been applied since the 1930s on airfoil sections to achieve lower skin friction drag. The principal types of active laminar-flow control are surface cooling and removal of a small amount of air from the boundary layer of suction. Generally, in surface injection, a secondary flow is injected from miniature openings or slots on the surface. In surface suction, the air is sucked from them. For highly swept wings, only suction can control sweep-induces cross-flow disturbances that promote boundary layer transition from laminar to turbulent ${ }^{[2-4]}$.

Flow control is a technology that offers the potential for improvements in aircraft fuel consumption. This broad area of research remains of great interest for its numerous potential benefits for both the military and civilian sectors. Improved manufacturing capabilities permit the general aviation industry to incorporate natural laminar flow in some of its aircraft designs for a chord Reynolds number less than 20 million. In active laminar flow control it is required to keep the flow laminar on the surface. In larger aircrafts or aircrafts with highly swept wings, this has not yet been applied to any operational case.

The objectives of flow control may lead to potential conflicts as the achievement of one particular goal may adversely affect another goal. The performance of an aircraft wing is measured by the liftto-drag ratio. Velocity profiles with higher velocity gradient are more resistant to transition and to separation but are associated with higher skin-friction

Corresponding Author: M. Ghaffarpour, Department of Mechanical, Engineering, University of Illinois at Chicago, 842 Taylor Street, 2039 ERF (MC 251), Chicago, Illinois 60607 
drag. Promoting transition will lead to a turbulent boundary layer that is more resistant to separation. By preventing separation, lift is enhanced and form drag is reduced. This will lead to increased lift according to a higher angle of attack. On the other hand, the viscous or skin-friction drag for a laminar boundary layer is an order of magnitude smaller than for a turbulent boundary layer. However, a laminar boundary layer is more prone to separation. This results in a loss in lift and an increase in form drag. Suppression of turbulence via active, energy-consuming control strategies is always possible, but the penalty for doing so often exceeds any potential savings. The objective is to achieve a desired effect with minimum energy consumption.

The simplest active flow control system is surface suction and injection. Suction and injection of a secondary airflow can have significant effects on the flow field. They affect particularly the shape of the velocity profile near the wall and change the boundary layer shape. An inflectional velocity profile can be produced by injection, adverse pressure-gradient or higher wall viscosity. Such profile is more prone to transition and to separation and is associated with lower, even negative, skin friction ${ }^{[5]}$.

The above-mentioned techniques are applied to the solid wall. There are some other techniques that are applied away from the wall. Some are as follows: Large-eddy breakup devices (also called outer-layer devices, or OLDs, acoustic waves bombarding a shear layer from outside ${ }^{[6]}$, additives introduced in the middle of a shear layer, manipulation of freestream turbulence levels and spectra, gust and magneto and electrohydrodynamic body forces ${ }^{[7]}$.

Another separation control technique is the application of direct synthetic jets (DJS). The concept involves low level periodic forcing to modulate the formation of vortices in separating flow. It applies acoustic streaming to form a synthetic jet. The exit neck is optimized for separation control ${ }^{[8]}$. Wynantskill ${ }^{[9]}$ has investigated the ability of local active flow control, on trailing edge. He has used the technique to control the wake generated downstream of a bluff trailing edge of a lifting airfoil. He has combined an active fliperone and synthetic jet and calculated the optimal frequency ratio between two actuators. Gilarranz et al. ${ }^{[10]}$ has designed compact, high-power synthetic jet actuators for flow separation control.

Available techniques to reduce skin-friction drag in turbulent wall-bounded flows include LEBUs and polymer $^{[11]}$. The first yields only modest drag reduction of the order of $10 \%$. The polymer additives result in substantial reduction of as much as $80 \%$. Polymers are appropriate only for hydrodynamic flows. They are occasionally utilized in practical pipelines, for example in the 800-mile Trans-Alaskan Pipeline System (TAPS). These techniques are not practical for external flows. Flow separation control is currently employed via vortex generators on the wings of most Boeing aircrafts. It is also used via blown flaps on older generation supersonic fighters or leading-edge extensions and strokes on newer generations ${ }^{[12-14]}$.

There are advances control systems such as feedback control system. Moin and Bewley ${ }^{[15]}$ have categorized reactive feedback control strategies. They categorized them by examining the extent to which they are based on the governing flow equations. Among them are adaptive and optimal control systems. An adaptive controller tries to optimize a specified performance index by providing a control signal to an actuator. In order to update its parameters, the controller thus requires feedback information relating to the effects of its control. The most recent innovation in adaptive flow control schemes involves the use of neural networks. However, hand tuning is required to achieve good convergence properties ${ }^{[16-18]}$.

In the optimal control method, feedback control laws are derived systematically for the most efficient distribution of control effort to achieve a desired goal. Abergel and Temam ${ }^{[19]}$ developed such optimal control theory for suppressing turbulence in a numerically simulated, two-dimensional Navier-Stokes flow. Their method requires full flow-field information, which is impractical. Choi et al. ${ }^{[20]}$ developed a more practical, wall-information only, control strategy. It is applied to the one-dimensional stochastic Burgers equation.

The purpose of this investigation is to study the effect of surface suction and injection on controlling the flow over a specific airfoil. The case studied is the flow field over a subsonic airfoil with suction and injection slots. The investigation is accomplished numerically. In order to study the effect of suction and injection, four slots are created in the airfoil suction side. Through these slots a secondary flow is injected into the main flow. In another test case a small amount of air is omitted from the main flow by suction.

\section{DESCRIPTION OF PHYSICAL PROBLEM}

The airfoil considered in this study is the Aerospatiale A-airfoil. The same airfoil is studied by Dahlstrom and Davidson ${ }^{[21]}$. Their experimental results have been used as a validation benchmark for the numerical solution presented here. The airfoil geometry, slot positions and dimensions are shown in Fig. 1. The chord length of the airfoil is $1026 \mathrm{~mm}$ and the thickness is $165 \mathrm{~mm}$. The width of each slot is $2.5 \mathrm{~mm}$ and the slots are $150 \mathrm{~mm}$ apart. In the case with suction slots, mass flow rate of air is $0.07 \mathrm{~kg} \mathrm{sec}^{-1}$ from each slot and in the injection case air is injected with the mass flow rate of $0.1 \mathrm{~kg} \mathrm{sec}^{-1}$ from each slot.

Five different angles of attack, 0, 5, 10, 13.3 and 20 are taken into consideration. The numerical solutions are conducted on three cases in each angle of attack. The three cases are airfoil with surface suction, surface injection and the base airfoil. The term base airfoil is referred to the airfoil without suction or injection. 


\section{THE COMPUTATIONAL METHODOLOGY}

The computational domain is consisted of the airfoil with 4 slots, on the suction side of the airfoil and an outer boundary. To minimize the disturbing effect of the airfoil on the outer boundary of the computational domain, it is set approximately 10 chord lengths away from the airfoil. Generated grid is a C-type mesh consisting 39'000 cells. In order to resolve the boundary layer, the mesh is refined on airfoil surface. A finer mesh is applied to the trailing edge wake region intentionally, which is obvious in Fig. 2. Over the suction/injection slots on the wall, the mesh is further refined throughout the boundary layer refined region as is shown in Fig. 3. Details of the refined mesh are shown in Fig. 4.

To solve the momentum and continuity equations the SIMPLE algorithm is used. The resulting system of the algebraic difference equations is solved in an uncoupled manner by the implicit procedure. A commercial CFD code, the FLUENT is used to solve the equations. The code is a finite volume NavierStokes solver.

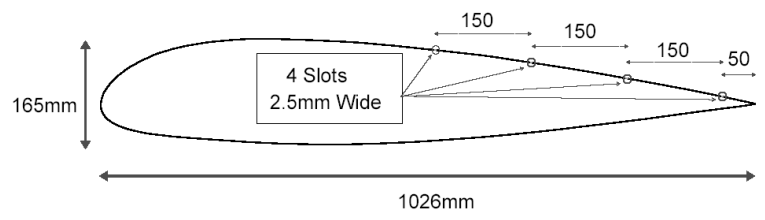

Fig. 1: Geometry of the airfoil and the location of the slots

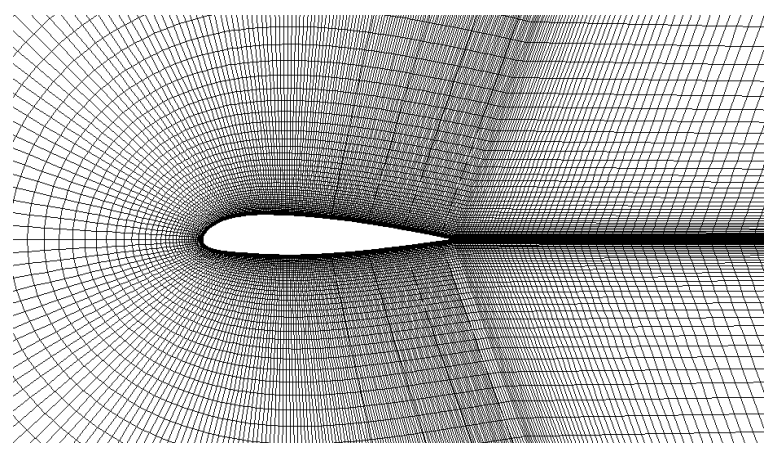

Fig. 2: The mesh around the airfoil is structured, consisted of 39'000 cells

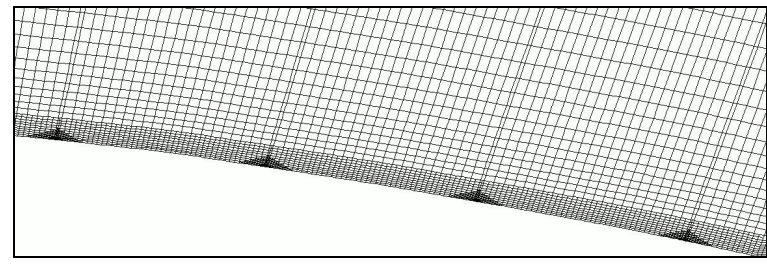

Fig. 3: Zoom of the refined mesh above the suction/injection slots
Air density is computed by the perfect-gas equation of state. Other properties of air are set to be constant. The Viscosity is $1.8 \mathrm{e}-5 \mathrm{Kg} \mathrm{ms}^{-1}$, thermal conductivity is $0.024 \mathrm{w} \mathrm{mK}^{-1}$ and the $\mathrm{Cp}$ is $1006 \mathrm{j} \mathrm{KgK}^{-1}$. At walls, the no-slip condition is applied. For boundary layer modeling the standard wall-function is used.
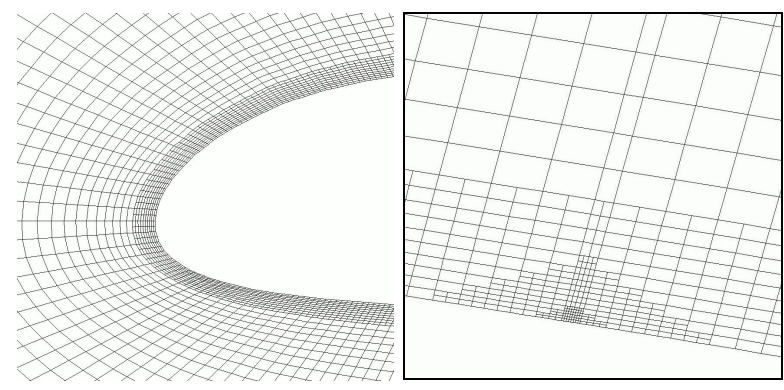

Fig. 4: Zoom of the refined mesh around the leading edge and above a suction/injection slot

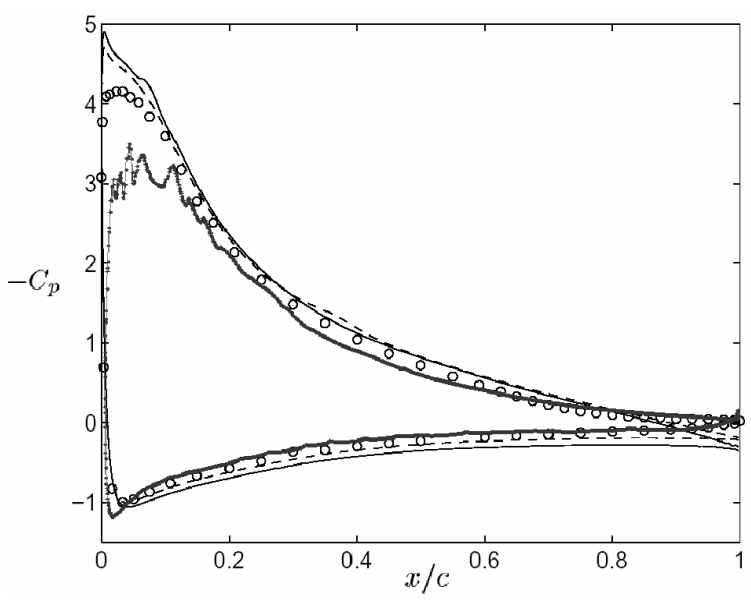

Fig. 5: Comparison of the present computation with experimental results. Bold line: present computation Fine solid and dashed lines: other numerical computations; circles: experimental results

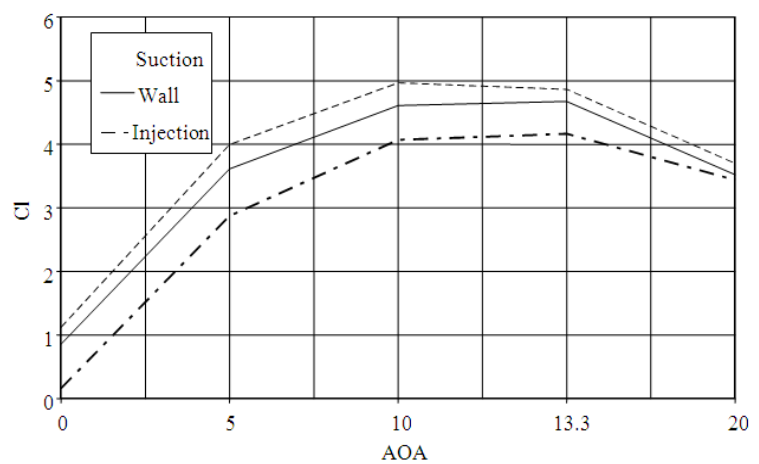

Fig. 6: Lift coefficient vs. AOA in different wall treatments 
The walls are considered as adiabatic boundaries. The Mach number is set to 0.15 . On the mesh outer boundary the temperature and the pressure are set to $300 \mathrm{~K}$ and $101 \mathrm{kPa}$, respectively. The properties of the air injected are the same as the main flow. For secondary airflow the turbulent kinetic energy and turbulent dissipation rate are set to be $1 \mathrm{~m}^{2} \mathrm{~s}^{-2}$ and $1 \mathrm{~m}^{2}$ $\mathrm{m}^{-3}$, respectively.

The viscous model used for modeling the turbulence is RNG $\mathrm{k}-\varepsilon$, with standard model constants. RNG $\mathrm{k}-\varepsilon$ equations have similar form as standard $\mathrm{k}-\varepsilon$ model equations except that the dissipation rate equation has an additional term related to the mean rate of strain and turbulence quantities that allows it to include more physics of the problem. Additionally, the model constants are derived from the Renormalization Group (RNG) theory as opposed to being empirically based.

\section{RESULTS}

Next step is to validate the model. This was accomplished through comparison with the experimental results of Dahlstrom and Davidson ${ }^{[21]}$. Their experimental results were available at angle of attack of 13.3 degrees and with base airfoil. As there was no solid model developed and no experimental results on hand to verify the results, the above mentioned reference is used as a validation source. Pressure coefficient is the characteristic, which is comparable. As Fig. 5 shows, the numerical and experimental results agree relatively well.

The effects of suction and injection on aerodynamic characteristics are investigated.

To investigate the effect of suction and injection, the results are compared with the base case. The following characteristics are compared for three cases: lift coefficient, friction coefficient and pressure drag coefficient. The most significant effect is in the lift. The results of lift coefficients for three cases are shown in Fig. 6. The advantage of the wing with suction slots is obvious in every tested angle of attack (AOA). In normal flight conditions, AOA of 5 and 10, suction presents a significant increase in lift coefficient equal to 10 and $6 \%$, respectively. Angle of attack of 13.3 degrees is a high lift condition in takeoff or landing. The lift coefficient in this angle of attack is increased by $4 \%$ due to surface suction. In AOA of 20 degrees the increase in lift coefficient is $5.5 \%$.

Injection has an unwanted effect on lift coefficient. It dramatically lowers the lift coefficient. In AOA of 5 degrees, injection lowers the lift coefficient by $23 \%$, which is the most significant among all cases. Injection also affects the surface friction. Figure 7 shows the suction and injection effects on surface friction in different angles of attack. Injection lowers the surface friction coefficient while decreasing the lift coefficient. Injection can be employed when lowering the surface friction is more important than increasing the lift. An engineering compromise is needed to choose the right wall treatment to reach the desired goal. Figure 8 shows the effect of suction and injection on pressure drag coefficient as is compared with the base case.

Figure 9 shows the pressure coefficient over the suction side of the airfoil in AOA of 20 degrees. Although the slots are placed near the trailing edge, they affect the upstream flow, as is shown in Fig. 9. So the total pressure over the airfoil surface is affected by the suction.

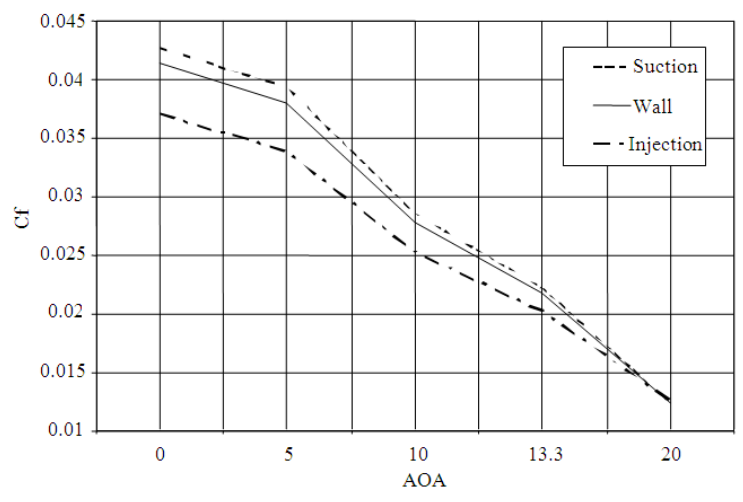

Fig. 7: Friction coefficient vs. AOA in different wall treatments

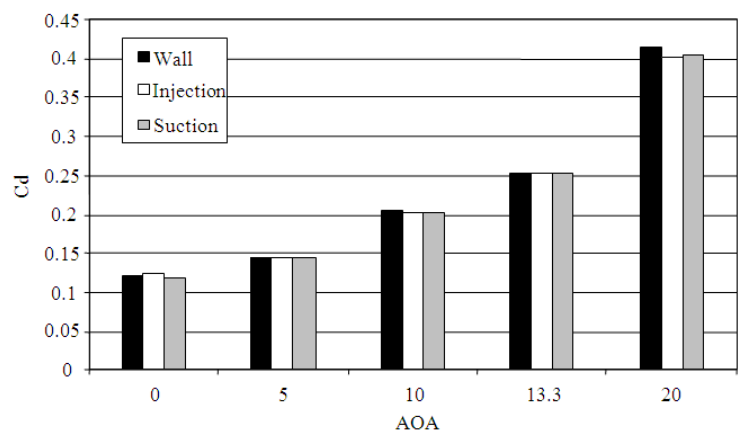

Fig. 8: Comparison of pressure drag coefficient with different wall treatments in five angles of attack

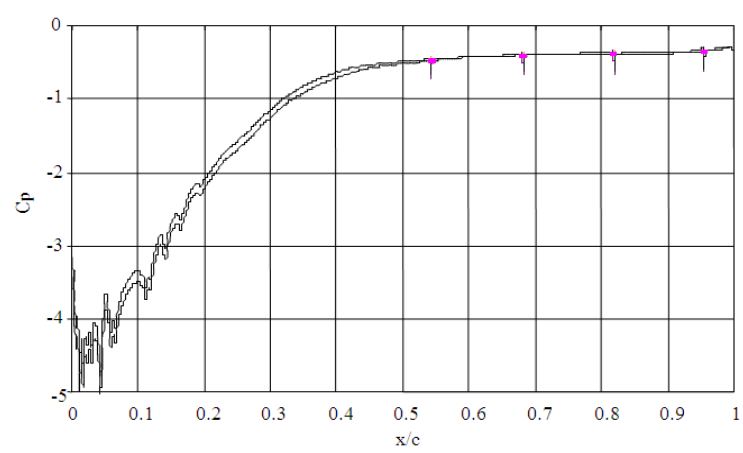

Fig. 9: Pressure coefficient on suction side of the airfoil in AOA of 20 degrees. Bold line: pressure coefficient without suction/injection; Fine line: pressure coefficient with suction 


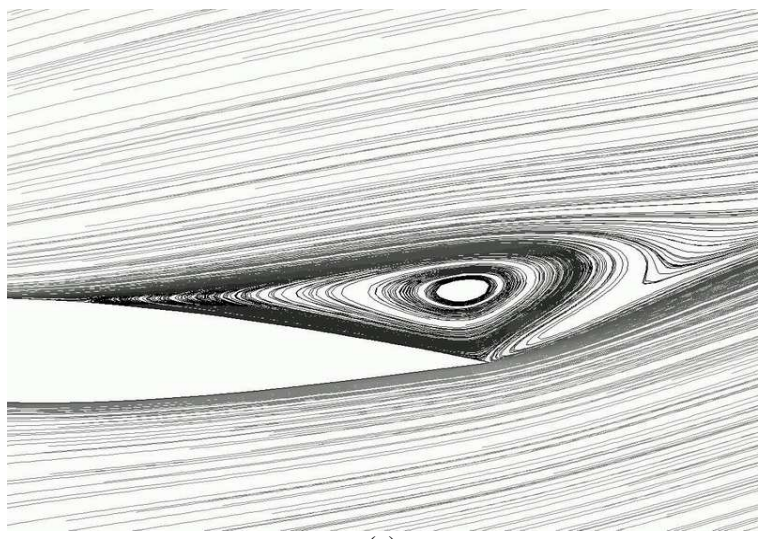

(a)

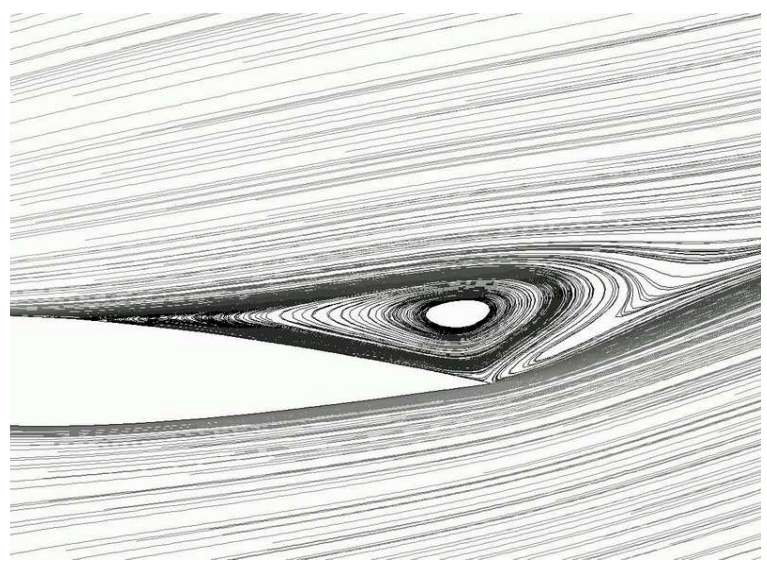

(b)

Fig. 10: Streamlines of the flow over the airfoil with AOA of 20 degrees Separation work is shown on the trailing edge. (a) Wall without section/injection (b) Wall with suction

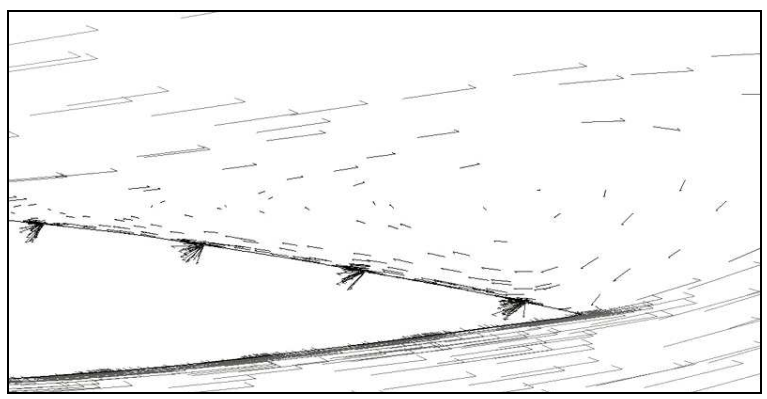

Fig. 11: Velocity vectors in a trailing edge wake in AOA of 20 degrees

Figure 10 shows the streamlines of two weeks of base airfoil (a) and suction case (b). The streamlines of the flow are shown. The results show that at the angle of attack of 20 degrees where we have separated flow, the given amount of suction actually cannot reattach the flow but it slightly reduces the size of the wake. The induced wake is too strong to be canceled by that magnitude of suction. Figure 11 shows the flow field in the separation region.

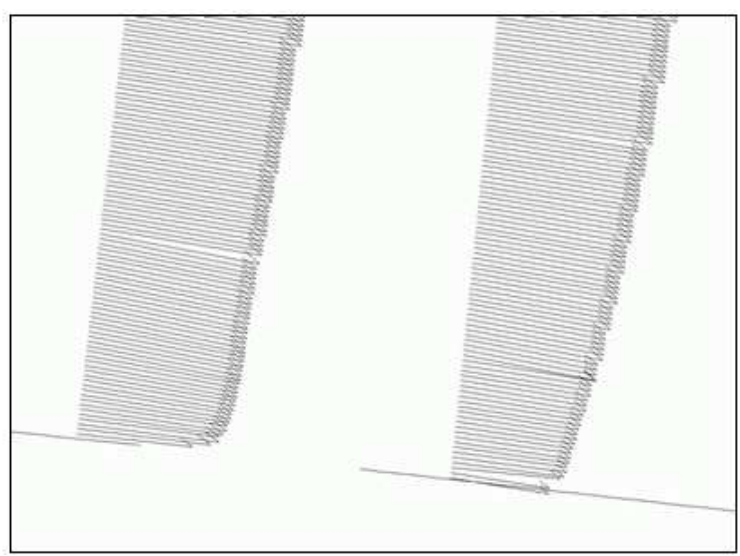

(a)

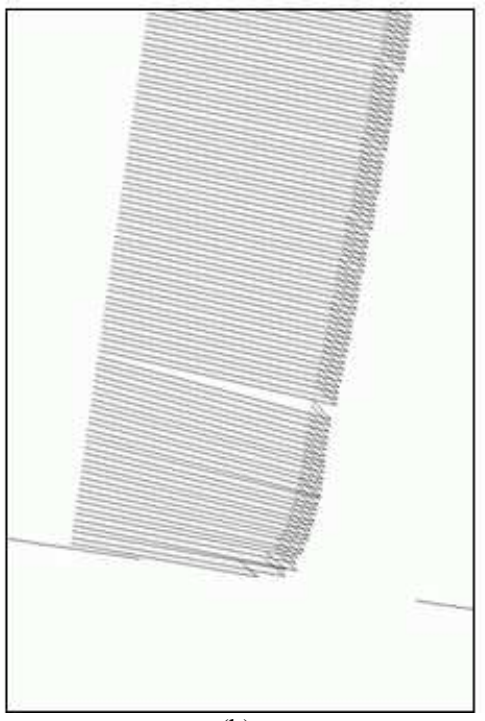

(b)

Fig. 12: Profiles in AOA of 10 degrees and wall suction (a) Boundary layer profiles before and after the first suction slot; (b) boundary layer profile before the second suction slot

The slots are actually sucking inside a reversed flow, which can somehow reduce the back-flow stream but cannot reverse the boundary layer profile.

The region that is actually involved in suction/injection process is the boundary layer.

To explain the effects of suction as mentioned above, we take a closer look at the boundary layer profiles. When the air is sucked into the wall the closest molecules and eddies to the wall are sucked in. It is obvious that the closer to the wall are the slowest. Therefore suction grabs the particles with minimum speed and the resulting profile lacks the low velocity particles in the bottom. It means that we artificially change the profile shape the way we desire. Exactly after the slot, the profile shape is sharper at the bottom. It means that the wall normal velocity gradient is higher and the shear stress is greater according to Stokes law. This results to higher skin friction. 


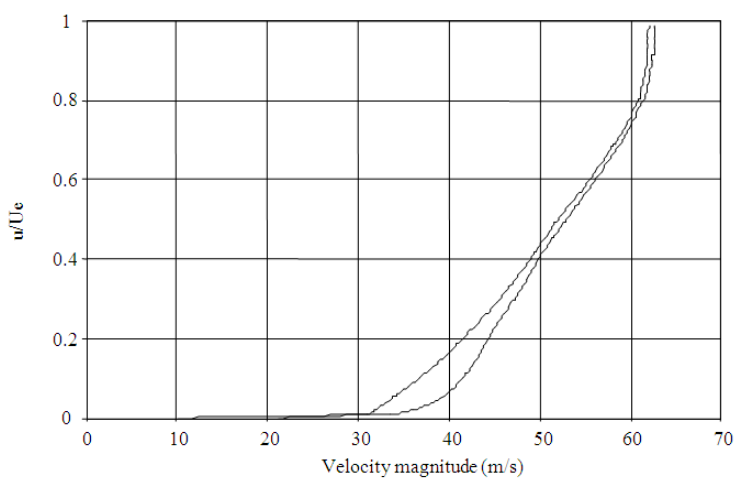

Fig. 13: Boundary layer profiles in AOA of 10 degrees with and without wall suction. Bold line: before the first suction slot; Fine line: after the first suction slot

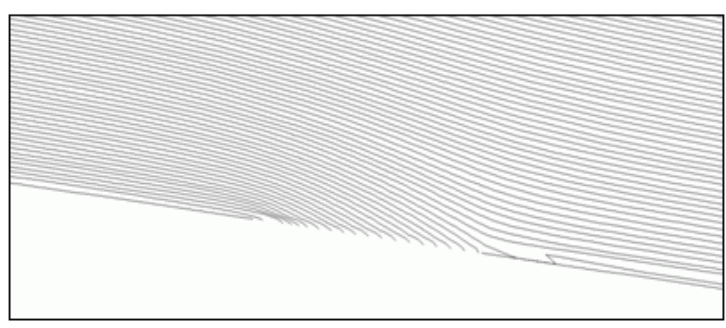

Fig. 14: Streamline of the flow adjacent to the solid boundary around first suction slot in AOA of 10 degrees

Figure 12 shows the boundary layer profiles before and after the first suction slot (a) and before the second suction slot (b). Under the influence of viscosity the boundary layer profile returns to its normal shape while the fluid travels on the solid surface. Before the second suction slot, the velocity profile is similar to the velocity profile before the first slot. The lower particles with greater velocity than what they have before give their energy to upper particles and the velocity profile is normalized again. Figure 13 shows the boundary layer profiles at AOA of 10 degrees with and without wall suction. The results show that the streamlines adjacent to solid are omitted from the field by being grabbed by the suction slots. Figure 14 shows a detailed view of streamlines near a suction slot.

\section{CONCLUSION}

A computational study has been accomplished to determine the effects of suction and injection in the aerodynamic characteristics of a specific airfoil. It is concluded that the suction slots near the trailing edge can significantly increase the lift coefficient and the injection decreases the skin friction. In the specific case studied here, at angle of attack of 5, which is correspondent to normal flight conditions, suction presents a significant increase in lift coefficient equal to $10 \%$.
This study can be a benchmark for the future numerical and experimental studies. The future work is to determine the optimal injection/suction value related to Mach number and angle of attack. Also the optimal number of slots and the space between them is a case of further studies. Note that this work is accomplished by assuming the slots a simple opening on the airfoil surface. In the future studies variety of opening shapes can be studied in order to design a more efficient slot opening shape.

\section{REFERENCES}

1. Braslow, A.L., 1999. A history of suction-type laminar-flow control with emphasis on flight research. American Institute of Aeronautics and Astronautics, Washington, D.C.

2. Thibert, J.J., J. Reneaux and V. Schmitt, 1990. ONERA activities for drag reduction. Proc. 17th Cong. Of the Intl. Council of the Aeronaut. Sci., 1: 1053-1064.

3. Wagner, R.D., M.C. Fischer, F.S. Collier Jr. and W. Pfenninger, 1990. Supersonic laminar flow control on commercial transports. Proc. 17th Cong. Of the Intl. Council of the Aeronaut. Sci., 1: 10731089.

4. Barnwell, R.W. and M.Y. Hussaini, (Eds.), 1992. Natural Laminar Flow and Laminar Flow Control. Springer-Verlag, New York.

5. Schetz, J.A., 1984. Foundation of Boundary Layer Theory for Momentum, Heat and Mass Transfer. Prentice Hall.

6. Apte, S. and V. Yang, 2002. Unsteady flow evolution in porous chamber with surface mass injection, Part 2: Acoustic excitation. AIAA J., 40: 2 .

7. Gad-el-Hak, M., Modern development in flow control. Appl. Mech. Rev., 49: 365-379.

8. Lorber, P., D. McCormick, T. Anderson, B. Wake, D. MacMartin Pollack, T. Corke and K. Breuer, 2000. Rotorcraft retreating blade stall control. AIAA, Fluids 2000 Conference and Exhibit, 19-22 Jun.

9. Wygnanski I. J., "The Control of Separation from Curved Surfaces and Blunt Trailing Edges" AFOSR GRANT F 49620-00-1-0070

10. McCormick, D.C., S.A. Lozyniak, D.G. MacMartin and P.F. Lorber, 2001. Compact highpower boundary layer separation control actuation development. Proc. 2001 ASME Fluids Engineering Division Summer Meeting, New Orleans, Louisiana, May, 29-Jun. 1.

11. Bushnell, D.M. and J.N. Hefner, (Ed), 1990. Viscous Drag Reduction in Boundary Layers, Progress in Astronautics and Aeronautics, vol. 123, American Institute of Aeronautics and Astronautics, Washington, D.C. 
12. Karim, M.A. and M. Acharya, 1994. Control of the dynamic-stall vortex over a pitching airfoil by leading-edge suction. AIAA J., 32: 1647-1655.

13. Alrefai, M. and M. Acharya, 1995. Controlled leading-edge suction for the management of unsteady separation over pitching airfoils. AIAA Paper No. 95-2188, Washington, D.C.

14. Roos, F.W., 1996. Microblowing for high-angleof-attack vortex flow control on a fighter aircraft. AIAA Paper No. 96-0543, Washington, D.C.

15. Moin, P. and T. Bewley, 1994. Feedback control of turbulence. Appl. Mech. Rev., 47: S3-S13.

16. Fan, X., L. Hofmann and T. Herbert, 1993. Active flow control with neural networks. AIAA Paper No. 93-3273, Washington, D.C.

17. Jacobson, S.A. and W.C. Reynolds, 1993. Active control of boundary layer wall shear stress using self-learning neural networks. AIAA Paper No. 933272, Washington, D.C.
18. Jacobson, S.A. and W.C. Reynolds, 1995. An experimental investigation towards the active control of turbulent boundary layers. Department of Mechanical Engineering Report No.

19. Abergel, F. and R. Temam, 1990. On some control problems in fluid mechanics. Theor. Comput. Fluid Dyn. 1: 303-325.

20. Choi, H., R. Temam, P. Moin and J. Kim, 1993. Feedback control of unsteady flow and its application to the stochastic burgers equation. J. Fluid Mech., 253: 509-543.

21. Dahlstrom, S. and L. Davidson, 2001. Large eddy simulation of the flow around an airfoil. AIAA 2001-0425, 39th AIAA Aerospace Sciences Meeting and Exhibit, 8-11 Jan., Reno, NV. 